\title{
Leveraging Diverse Propagation and Context for Multi-Modal Vehicular Applications
}

\author{
Pengfei Cui, Hui Liu, Jialin He, Onur Altintas, Rama Vuyyuru, Dinesh Rajan, and Joseph Camp
}

\begin{abstract}
Vehicular wireless channels have a high degree of variability, presenting a challenge for vehicles and infrastructure to remain connected. The emergence of the white space bands for data usage enables increased flexibility for vehicular networks with distinct propagation characteristics across frequency bands from $450 \mathrm{MHz}$ to $6 \mathrm{GHz}$. Since wireless propagation largely depends on the environment in operation, a historical understanding of the frequency bands' performance in a given environment could expedite band selection as vehicles transition across diverse scenarios. In this paper, we leverage knowledge of in-situ operation across frequency bands with real-time measurements of the activity level to select the the band with the highest throughput. To do so, we perform a number of experiments in typical vehicular topologies. With two models based on machine learning algorithms and an in-situ training set, we predict the throughput based on: $(i$.) prior performance for similar context information (e.g., SNR, GPS, relative speed, and link distance), and (ii.) real-time activity level and relative channel quality per band. In the field, we show that training on a repeatable route with these machine learning techniques can yield vast performance improvements from prior schemes.
\end{abstract}

\section{INTRODUCTION}

Drivers and passengers around the world could utilize a wide array of vehicular applications ranging from real-time traffic monitoring and safety applications to infotainment applications spanning news, weather, audio, and video streams. However, the continuous use of such applications is limited due to the challenge of transmitting over highly-dynamic vehicular wireless channels. In such networks, the increasing availability of different frequency bands with correspondingly diverse propagation characteristics could allow flexibility and robustness of vehicular links. Even with spectral flexibility, links are extremely tenuous, demanding instantaneous decisions to remain connected, motivating an algorithm that can find the appropriate frequency band quickly and according to the current environmental context.

Cognitive radio mechanisms which interleave channel accesses also motivate the frequency band selection problem of finding the optimal spectrum on which to transmit [1]. Prior work has considered a number of challenges in leveraging white space frequencies including spectrum sensing, frequency-agile operation, geolocation, solving stringent spectral mask requirements, and providing reliable service in unlicensed and dynamically changing spectrum [2]. In particular, there has recently been an acceleration in spectrum sensing work [3]-[5]. Based on these works, protocols have been built for multi-channel and/or multiband wireless operation [6]-[8].

P. Cui, H. Liu, J. He, D. Rajan, and J. Camp are with Southern Methodist University, Dallas, TX. O. Altintas is with Toyota InfoTechnology Center, Co. Ltd., Tokyo, Japan. R. Vuyyuru is with Toyota InfoTechnology Center, U.S.A. Inc., Mountain View, CA. The SMU authors were supported on this work by NSF grants CNS-0958436, CNS-1040429, and CNS-1150215.
Other works have presented methods for searching for the most efficient transmission channel [9], discovering channel information [3], [8], and estimating channel quality [6]. Finally, the emergence of a number of diverse sensors on a vehicle motivates work on heterogeneous wireless networks, which have different frequency bands and technologies [10]. Thus, the various communication standards have diverse throughput capacity, allowing the choice of technology to possibly usurp frequency band decisions. For example, an 802.11n link at $5.8 \mathrm{GHz}$ with high levels of loss might still be a better choice than a Bluetooth link at $2.4 \mathrm{GHz}$ with little loss due to the discrepancy of hundreds of Mbps in throughput capacity.

However, for the purposes of this work, we assume the underlying technology is the same to evaluate the choice of frequency band. While these works have considered spectral activity and developing protocols and algorithms to find spectral holes, less of a focus has been on coupling such information with historical performance in a given propagation environment. In this paper, we develop multiband adaptation protocols which couple the prior knowledge of in-situ performance of various bands with the instantaneous knowledge of spectral activity, SNR, and current location of each band to arrive at a decision on the optimal band to transmit. To do so, we use an off-the-shelf platform that allows direct comparison and simultaneous experimentation across four different wireless frequency bands from $450 \mathrm{MHz}$ to $5.8 \mathrm{GHz}$ with the same physical and media access layers.

The main contributions of our work are as follows:

- We first develop a framework for multiband adaptation using both historical information and instantaneous measurements. This framework is broad enough to study adaptation across licensed and unlicensed bands, including white space frequency bands.

- We propose two different machine-learning-based adaptive algorithms. The first machine learning algorithm, which we refer to as the Location-based Look-up Algorithm, is based on the idea of $k$-nearest-neighbor classification. The second machine-learning-based algorithm uses decision trees for classification. For comparison, we also create two baseline adaptation algorithms which attempt to make the optimal band selection based on only: (i.) historical performance data, and (ii.) instantaneous SNR measurements across various bands.

- We perform extensive outdoor $\mathrm{V}-2-\mathrm{V}$ experiments to evaluate the proposed algorithms. Our results indicate that the proposed machine learning algorithms improve throughput by up to $49.3 \%$ over these baseline methods. 


\section{Multiband Adaptation}

In this section, we first formulate the multiband adaptation problem in vehicular wireless links and introduce the set of information we use to make multiband decisions, which we refer to as context information. We then propose two machinelearning-based multiband adaptation algorithms for vehicular channels. For comparison, we also propose two baseline adaptation methods based on existing solutions which consider historical and instantaneous information independently.

\section{A. Problem Formulation}

Consider a system with $n$ frequency bands, represented by an index set $\{1,2, \ldots, n\}$. The objective is to select the optimal band, $b_{\text {best }}$, to transmit at each time instant that maximizes a desired objective function such as throughput. The throughput $r_{i}$ on band $i$ depends on several factors such as received signal power $P_{R}^{i}$, noise power $P_{N}^{i}$, the channel busy time $B^{i}$, the velocity of the transmitter, $v_{t x}$, the velocity of the receiver, $v_{r x}$, and location information which depends on each algorithm and will be specified in the algorithm section. The aforementioned set of all information used to make multiband decisions composes the users context. This relationship is represented in general as $r_{i}=f\left(P_{R}^{i}, P_{N}^{i}, B^{i}, v_{t x}, v_{r x}\right.$,context information per algorithm). The objective can be stated as:

$$
b_{\text {best }}=\arg \max _{i} r_{i}
$$

The framework allows separation of the interference from other nodes using the same technology (via the busy time $B^{i}$ ) and other technologies (via the noise level $P_{N}^{i}$ ). For instance, an 802.11 node can decode the packets of other 802.11 nodes but can only sense instantaneous noise levels from ZigBee/Bluetooth nodes. Decoding the packets can provide increased knowledge such as data rate and packet size to determine the duration of the channel use. We can exploit the long-term behavior by using historical performance data for the collected context information (e.g., $v_{t x}, v_{r x}, B^{i}, P_{N}^{i}$, $\left.P_{R}^{i}\right)$ [11].

To represent the utilization level of the channel, we define busy time, $B$, as the percentage of time when the channel is occupied by all competing sources $x_{j}(j=1,2,3, \ldots)$ other than the intended transmitter $y$. For 802.11-based transmissions, the busy time on band $i$ is defined as:

$$
B^{i}=\frac{\sum_{j} \sum_{k} \frac{L_{k}^{x_{j}}}{R_{k}^{x_{j}}}}{\sum_{k} \frac{L_{k}^{y}}{R_{k}^{y}}+\sum_{j} \sum_{k} \frac{L_{k}^{x_{j}}}{R_{k}^{x_{j}}}+S \sigma}
$$

where $L_{k}^{x_{j}}$ and $R_{k}^{x_{j}}$ represent the packet length in bits and data rate at which that packet is transmitted, for external sources $x_{j} ; S$ and $\sigma$ are the number of idle slots and slot duration, respectively. When considering the activity level of non-802.11 users (e.g., the bands currently licensed to TV and other non802.11 devices), we use the received signal level from non802.11 interfering sources $P_{N}^{i}$ on band $i$ directly as an input to our algorithms.

\section{B. Multiband Adaptation Algorithms}

In order to evaluate the proposed multiband adaptation algorithms, we construct two baseline methods: (i.) We search for the most commonly selected band as the best band in the historical data and choose it as the final band decision. (ii.) For each band, we build a lookup table for throughput $T_{\text {ideal }}$ in an idealized channel given the RSSI and obtain the best band according to following:

$$
\max _{i} T_{\text {ideal }}^{i} \times\left(1-B^{i}\right),
$$

The throughput $T_{\text {ideal }}$ is measured with an Azimuth ACE-MX channel emulator [12]. The details of the system setup and data collection are discussed in Section III.

Machine learning has been used as an important tool in wireless communications [13]. When a user enters an area, the machine learning algorithm can learn from the historical data and train to select the potential optimal band given the input, e.g., $P_{R}^{i}, v$ and $P_{N}^{i}$. We propose two multiband adaptation algorithms based on two machine learning methods: k-nearest neighbor (KNN) and decision trees.

Location-based Look-up Algorithm. KNN is a machine learning method based on searching for the closest training data points in the feature space and various modified versions have been applied successfully for classification [14]. In the Location-based Look-up Algorithm, we search for the closest neighbors of a testing point by using each parameter one by one in the input set. The Location-based Look-up Algorithm additionally involves geographic information for band selection other than received signal power $P_{R}^{i}$, noise power $P_{N}^{i}$, the activity/occupancy level $B^{i}$, the velocity of the transmitter, $v_{t x}$. The performance of the selected training data points is averaged to generate an estimate of the performance at each band. Then the band with the highest throughput performance is selected as $b_{\text {best }}$.

For the Location-based Look-up Algorithm, context information involves location information $g$ which is the GPS latitude and longitude, $v, P_{R}^{i}, P_{N}^{i}$ and $B^{i}$. To make a band prediction, we have four look-up blocks to reduce the training data points which are similar to the testing data point. First, we search for the historical data which is closest to the testing data based on GPS location. If the number of found historical data points is less than a predefined threshold, $\theta_{\text {AArea }}$, we increase the distance range (the actual threshold is discussed in Section III). Then, based on the filtered historical data, we collect $\theta_{\text {AArea }}$ data points which are closest to $P_{R}^{i}$, where $\theta_{\text {AArea }}$ is the threshold of the number of collected data points. A similar process is repeated based on $P_{N}^{i}$ and $v$, respectively. After deciding the final data set, we average the throughput of data points at each band. The key steps of this algorithm are presented as Algorithm 1.

Region-based Decision Tree Algorithm. Decision trees are a widely-used machine learning algorithm due to their low complexity and stable performance [15]. A decision tree can model the relationship in the training data between the context information and the optimal band as a set of tree-like deduction structures. Before implementing the training process, we prepare a training set including a group of training data points of $\left\{v_{t x}, v_{r x}, P_{R}^{1}, \ldots, P_{R}^{n}, B^{1}, \ldots, B^{n}, P_{N}^{1}, \ldots, P_{N}^{n}, b_{\text {best }}\right\}$ based on the collected measurements. We obtain $b_{\text {best }}$ by 


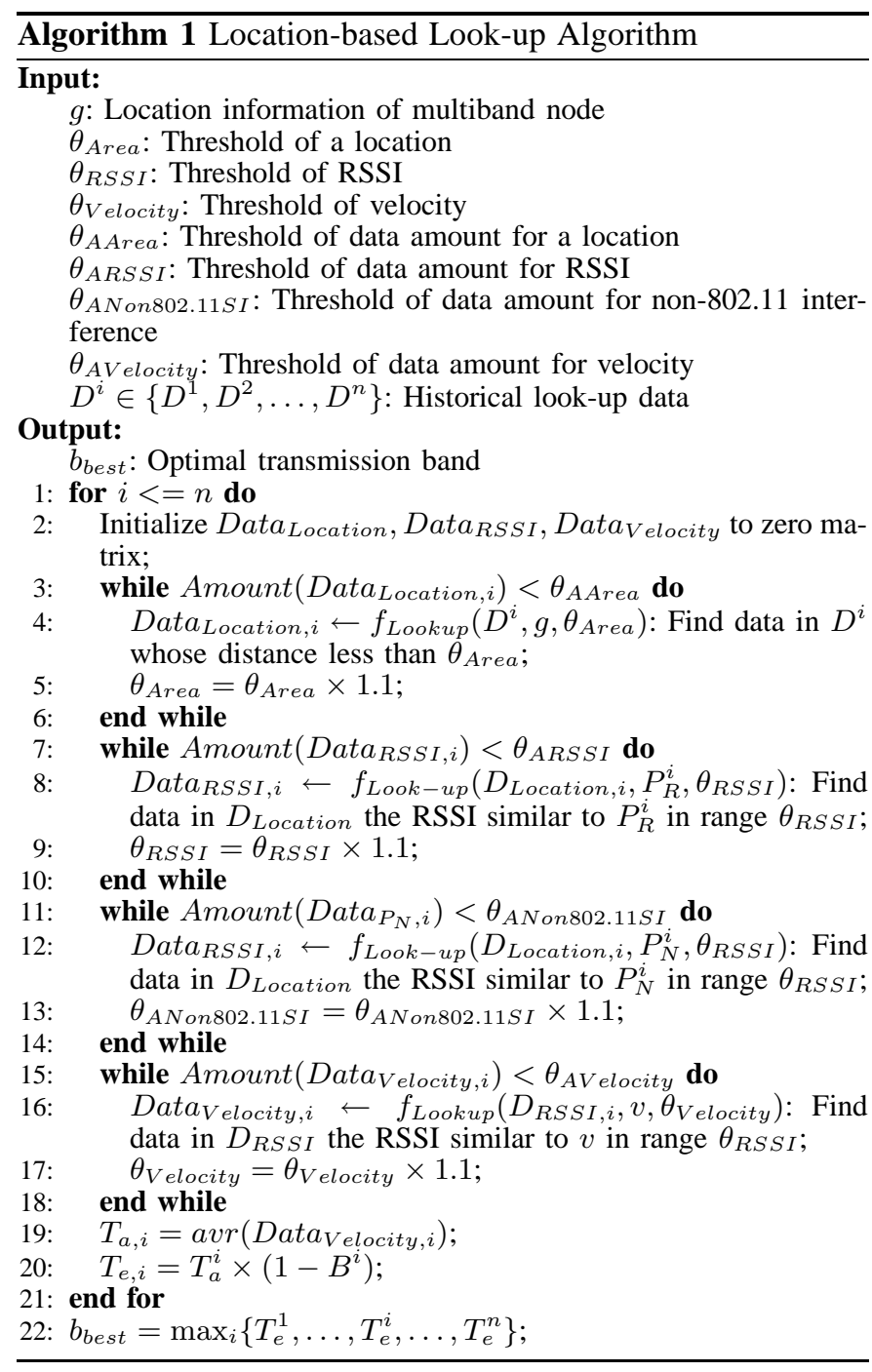

comparing the throughput performance of all available bands and selecting the band with the highest throughput. We choose the C4.5 algorithm to generate our decision tree [16], a widely-used algorithm based on the information entropy gain. At each intermediate node in the decision tree, the learning algorithm calculates the information entropy gain of splitting the remaining training data points based on each parameter in the input set, e.g., $P_{R}^{i}, v$ or $P_{N}^{i}$. Then, it compares and selects the parameter with the highest entropy gain to decide the test condition at each intermediate node until all training data points are classified. The leaf nodes indicate the optimal band for prediction in our application. Then, the trained decision structure is integrated into the transmitter protocol stack. With the collected context information, the decision structure can suggest the band with the best throughput performance.

The relationship between the context information and the best band could differ at different locations because of diverse propagation environment characteristics. To reduce the heterogeneity of training data from different locations, we split the vehicular route into several regions and implement the training process based on the historical data collected in each region. Then, the trained decision structure is integrated in our system

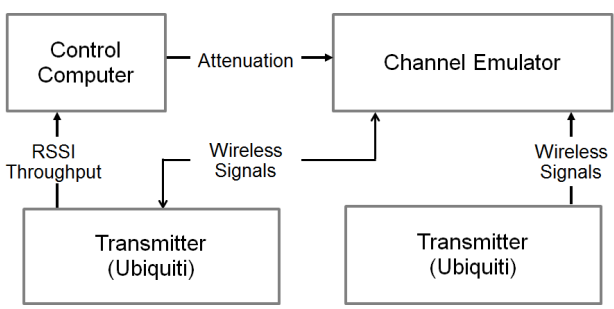

Fig. 1. Experimental setup for channel emulator.

for multiband adaptation in each region. The granularity of regional division is one parameter that affects the training set as well as the performance of the resulting decision tree. We evaluate the granularity of these divisions in Section III.

\section{EXPERIMENTS FOR MULTIBAND AlgORITHMS}

To study these algorithms, we have developed indoor and in-field experiments on an off-the-shelf wireless platform. To ensure the results are broadly applicable, we employ a Linuxbased 802.11 testbed [17]. The platform includes a Gateworks 2358 motherboard with Ubiquiti XR radios (XR9 at $900 \mathrm{MHz}$, XR2 at $2.4 \mathrm{GHz}, \mathrm{XR} 5$ at $5.2 \mathrm{GHz}$ ) and a DoodleLabs DL475 radio at $450 \mathrm{MHz}$ [18], [19]. We use an Azimuth ACEMX channel emulator for controllable propagation and fading characteristics with a broad range of industry-standard channel models from $450 \mathrm{MHz}$ to $5.9 \mathrm{GHz}$ [12].

\section{A. In-lab Experiments for Radio Characterization}

To establish an SNR-to-throughput relationship for the SNRbased Throughput Look-up Algorithm, we use an experimental setup where two wireless nodes communicate across repeatable emulated channels generated by Azimuth ACE-MX channel emulator (Figure 1). For a given band and card, we measure the throughput of a fully-backlogged UDP flow using the iperf traffic generator. We use constant attenuation over an idealized channel condition and repeat the experiment to produce various RSSI values. Despite the same physical and media access control layers of the radios, there are slight differences in the throughput achieved per radio at the same attenuation level. Thus, we normalize these throughput values to have the same maximum throughput across radio types for a fair comparison of the frequency bands.

\section{B. Experimental Design for In-field Data Collection}

We now describe the in-field experimental design to obtain a data set for evaluating our multiband algorithms. Two Gateworks boards, each containing the aforementioned four radios, are installed on two cars. One node is always the receiver and at a fixed location. The other node is always the transmitter and travels around the block of a public park as shown in Figure 2. One loop of the route will be used as a unit of training in the next section.

During each loop, the transmitter sends a fully-backlogged UDP flow using iperf on each of the four radios simultaneously. To focus on band selection and ensure the greatest range, we disable autorate and use a fixed data rate of $6 \mathrm{Mbps}$. The receiver continually performs a tcpdump of all received 802.11 packets [20]. Additionally, a QH 400 Quad Ridge Horn Antenna (shown in Figure 2) is connected to a Rhode \& Schwarz FSH8 mobile spectrum analyzer at the receiver to monitor spectral activity. Then, based on the time stamps, we 

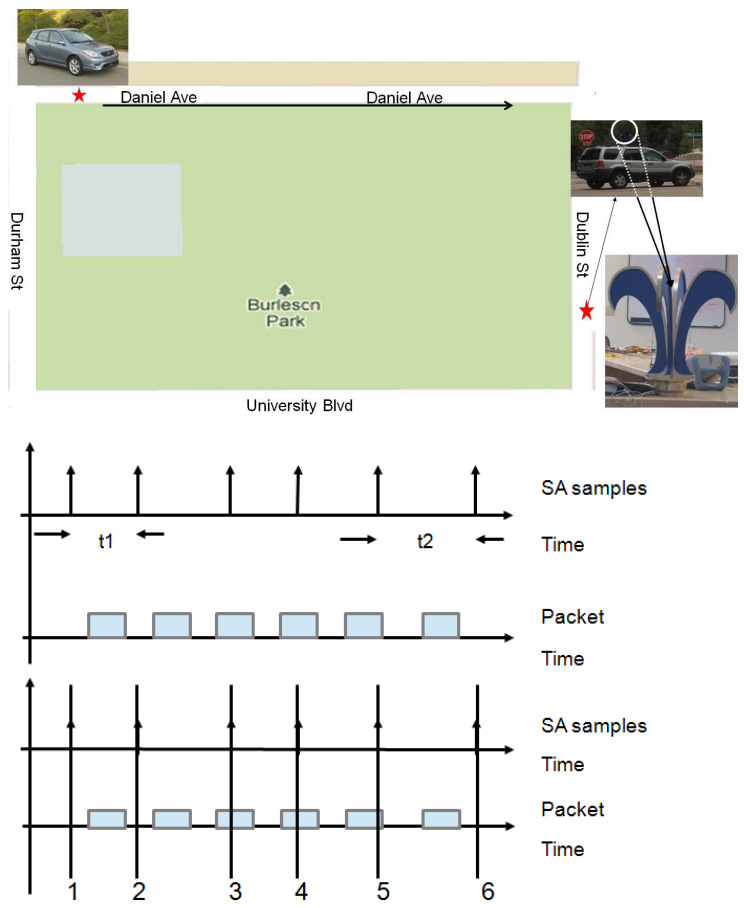

Fig. 3. Spectrum Analyzer Data Processing.

remove 802.11 packets from the spectral trace so that only non-802.11 interference will contribute to $P_{N}^{N}$.

Figure 3 shows how we obtain the non-802.11 interference, $P_{N}^{i}$. We expunge the spectrum analyzer (SA) samples which overlap in time with the dumped 802.11 packets, such as packets 3, 4, and 5. Then, the reported interference value will not contain the received power from 802.11 packets, which have already been considered via the busy time, $B$.

The in-field data is processed offline where data from all instruments involved is synchronized based on the GPS time stamps. As discussed in Section III-A, the throughput of each radio is normalized based upon emulator experiments to account for any manufacturing differences.

\section{Performance Analysis of Algorithms}

We now investigate the performance of our proposed algorithms with the experimental setup described in Sections III-A and III-B. The metrics of Accuracy and Throughput Gap are used in the evaluation. We consider each second of the in-field trace and observe the frequency band that had the highest throughput. The Accuracy is defined as the percentage of best band predictions match the observed best band, where a prediction is made each second. Conversely, the Throughput Gap is defined as the difference between the throughput observed on the best band and the throughput achieved by the predicted best band over the throughput of the observed best band. In the situation where the exact best band is not chosen, the throughput could be close between the chosen band and the optimal band, meaning that the incorrect band choice did not result in a large throughput loss. Thus, the Throughput Gap metric captures the severity of the incorrect choice.

Since the SNR-based Throughput Look-up Algorithm requires only emulator-based training, the Accuracy and Throughput Gap can be calculated for all loops of the infield trace. However, the Location-based Look-up Algorithm

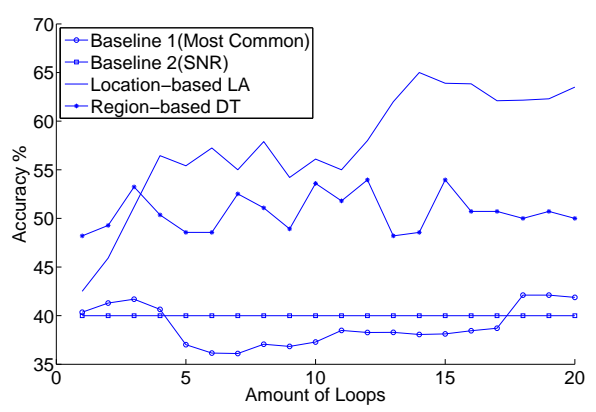

Fig. 4. Accuracy of the four multiband algorithms.

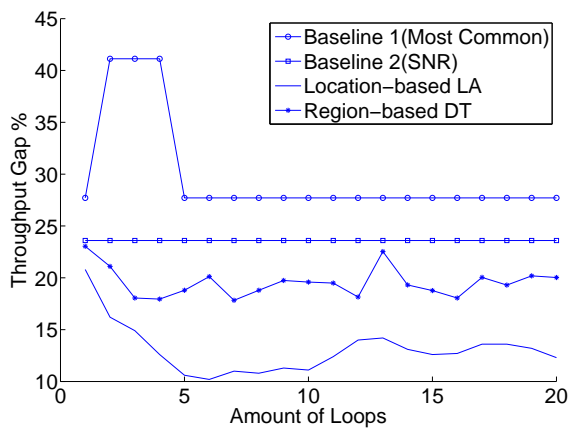

Fig. 5. Throughput Gap of the four multiband algorithms.

and the Region-based Decision Tree Algorithm requires in-field training. Thus, the data set must be divided into a training set and testing set for evaluation.

In Figure 4, we show the aforementioned Accuracy of the four multiband algorithms in selecting the band with the highest throughput. The $\mathrm{x}$-axis represents the number of loops around the block of the mobile transmitter (shown in Figure 2) that will be used by the machine-learningbased algorithms. We use the same training and testing set to compare the Location-based Look-up Algorithm and the Region-based Decision Tree Algorithm. From the results, we observe the following:

- At each loop, the first baseline algorithm, Most Commonly-Selected Band, uses the band with the greatest long-term average of the percentage of time that band yields the highest throughput over the previous loops. The accuracy ranges from $36.1 \%$ to $42.9 \%$.

- The second baseline algorithm, SNR-based Throughput Look-up, maintains a $39.2 \%$ across all the data since it relies only on emulator-based training.

- The Region-based Decision Tree Algorithm has an accuracy ranging from $48.2 \%$ to $54.0 \%$ but contains many dips due to the relationship between the context information and the distribution of the best band choice changing on a loop-by-loop basis. Additional training data slightly improves the decision structure overall but primarily induces additional noise in the training process.

- The Location-based Look-up Algorithm begins with an accuracy of $42.5 \%$ but improves the most out of any algorithm to finish with an accuracy $62.5 \%$ with a highest accuracy of $65.0 \%$ occurring after loop 14. Additional in-field training loops are likely to further improve the multiband selection accuracy.

Figure 5, depicts the Throughput Gap of the four algorithms 


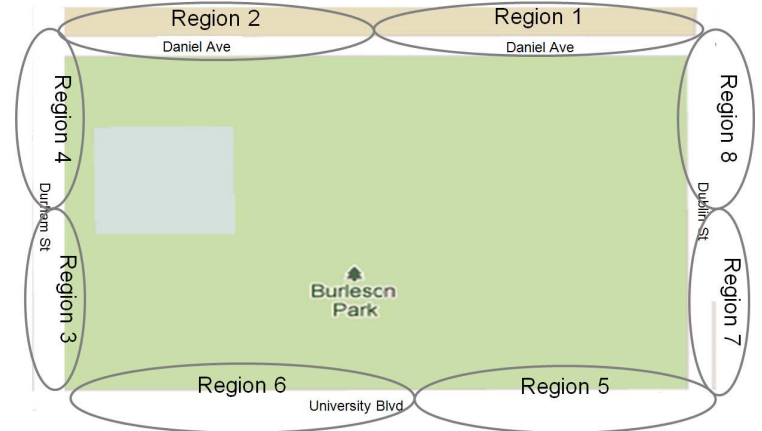

Fig. 6. Spatially splitting experimental area into 8 regions.

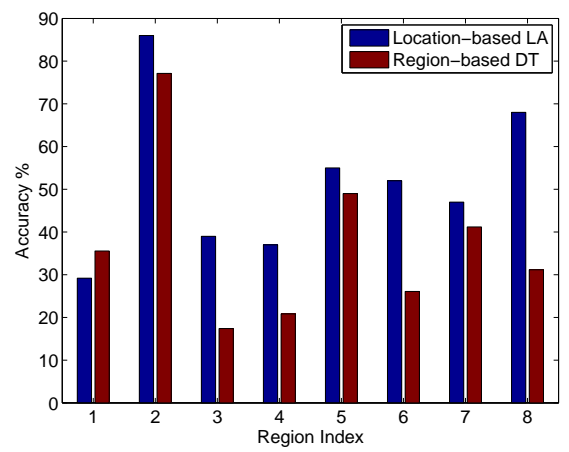

Fig. 7. Accuracy when dividing training set into 8 regions.

we evaluated and shows the following.

- The Most Commonly-Selected Band Algorithm has two different modes of throughput gap based upon which band has the highest long-term percentage. For loops 2-4, the choice is $5.8 \mathrm{GHz}$, which has a gap of $41.1 \%$ using the test set. For all other loops, the choice is $2.4 \mathrm{GHz}$, which has a gap of $27.7 \%$.

- The SNR-based Throughput Look-up Algorithm shows a baseline performance of $23.6 \%$ for the throughput gap.

- The Region-based Decision Tree Algorithm benefits from additional training, going from a throughput gap of $23.0 \%$ to $20.0 \%$. Spatial and temporal changes to context information bring dips to the curve.

- Finally, the Location-based Look-up Algorithm takes only 6 loops of training to reach its lowest value of $10.2 \%$ in terms of throughput gap. From loop 1 to loop 20, the throughput gap goes from $20.8 \%$ to $12.3 \%$, which might still be improved upon with additional training.

We now consider the effect of further sub-dividing infield experimental testing data into regions for our Locationbased Look-up Algorithm and Region-Based Decision Tree Algorithm. To do so, we divide the loop around the park into eight regions as shown in Figure 6, which has two competing effects: (i.) Smaller regions allow similar experimental data to be used in the training process, potentially improving the decision structure. (ii.) For a given training set, dividing it into regions reduces the number of training points for the machine learning algorithms, potentially weakening the decision structure. In Figure 7, we observe the Accuracy of the eight regions for both algorithms.

- In all but Region 1, the Location-based Look-up Algorithm has better performance than Region-based Decision Tree Algorithm. The improved accuracy of the former algorithm can be attributed to its ability to distinguish between the middle of a given region to the boundary, considering each point differently. For the Region-based Decision Tree Algorithm to capture such a notion, the regions would have to be further sub-divided, increasing the number of trees and reducing the training set per tree.

- For this training set, the reduction in training data caused by the regional divisions had a net loss on the performance of the Region-based Decision Tree Algorithm. However, if the training set was much larger for a given area, the net effect of regional divisions could be positive.

\section{CONCLUSION}

In this paper, we investigated multiband adaptation to leverage the propagation and context for vehicular applications. We did so by proposing two machine-learning-based schemes and compared their performance against two baseline schemes. In our experimental analysis, we evaluated the performance of these algorithms in the field on an off-the-shelf platform. Experimental results demonstrate that the proposed algorithms can achieve up to $49.3 \%$ greater throughput than the baseline algorithms with an accuracy up to $65 \%$. In future work, we will study the impact that multiple diverse environments have on the training as well as evaluate the optimal use of multiple, diverse radios in unison.

\section{REFERENCES}

[1] A. Ghasemi and E. Sousa, "Spectrum sensing in cognitive radio networks: requirements, challenges and design trade-offs," Communications Magazine, IEEE, vol. 46, no. 4, pp. 32-39, 2008.

[2] S. Shellhammer, A. Sadek, and W. Zhang, "Technical challenges for cognitive radio in the tv white space spectrum," in IEEE ITA Workshop, 2009, pp. 323-333.

[3] S. Rayanchu, V. Shrivastava, S. Banerjee, and R. Chandra, "Fluid: improving throughputs in enterprise wireless lans through flexible channelization," in ACM MobiCom 2011, pp. 1-12.

[4] S. Kim, H. Bertoni, and M. Stern, "Pulse propagation characteristics at $2.4 \mathrm{ghz}$ inside buildings," Vehicular Technology, IEEE Transactions on, vol. 45 , no. 3, pp. 579-592, 1996.

[5] D. Cabric, S. Mishra, and R. Brodersen, "Implementation issues in spectrum sensing for cognitive radios," in IEEE 38th ASILOMAR 2004. vol. 1, pp. 772-776.

[6] V. Kanodia, A. Sabharwal, and E. Knightly, "MOAR: A multi-channel opportunistic auto-rate media access protocol for ad hoc networks," in Broadnets, San Jose, CA, October 2004.

[7] D. Raychaudhuri and X. Jing, "A spectrum etiquette protocol for efficient coordination of radio devices in unlicensed bands," in IEEE PIMRC 2003., vol. 1, pp. 172-176.

[8] A. Sabharwal, A. Khoshnevis, and E. Knightly, "Opportunistic spectral usage: Bounds and a multi-band csma/ca protocol," Networking, IEEE/ACM Transactions on, vol. 15, no. 3, pp. 533-545, 2007.

[9] J. Mo, H. So, and J. Walrand, "Comparison of multi-channel mac protocols," in ACM 8th WSWIM,2005, pp. 209-218.

[10] E. Hossain, G. Chow, V. Leung, R. McLeod, J. Mišić, V. Wong, and O. Yang, "Vehicular telematics over heterogeneous wireless networks: A survey," Computer Communications, vol. 33, no. 7, pp. 775-793, 2010.

[11] R. Meikle and J. Camp, "A global measurement study of context-based propagation and user mobility," in ACM 4th HotPlanet,2012, pp. 21-26.

[12] "Aximuth ACE-MIMO Channel Emulator," http://www.azimuthsystems.com, Mar. 2011.

[13] S. Haykin, "Cognitive radio: brain-empowered wireless communications," Selected Areas in Communications, IEEE Journal on, vol. 23, no. 2, pp. 201-220, 2005.

[14] H. Zhang, A. Berg, M. Maire, and J. Malik, "Svm-knn: Discriminative nearest neighbor classification for visual category recognition," in IEEE CVPR 2006, vol. 2, pp. 2126-2136.

[15] R. Banfield, L. Hall, K. Bowyer, W. Kegelmeyer, et al., "A comparison of decision tree ensemble creation techniques," IEEE Trans on Pattern Analysis and Machine Intelligence, vol. 29, no. 1, p. 173, 2007. 
[16] M. Hall, E. Frank, G. Holmes, B. Pfahringer, P. Reutemann, and I. Witten, "The weka data mining software: an update," ACM SIGKDD Explorations Newsletter, vol. 11, no. 1, pp. 10-18, 2009.

[17] "Openwrt wireless freedom," https://openwrt.org/, Dec. 2011.

[18] "Cambria GW2358-4," http://www.gateworks.com/, 2007.

[19] "Ubiquiti xtremerange series of radio," http://www.ubnt.com/, 2012.

[20] V. Jacobson, C. Leres, and S. McCanne, "The tcpdump manual page," Lawrence Berkeley Laboratory, Berkeley, CA, 1989. 